\title{
Components of NOTCH Signaling for Uterine Cancer Patients' Prognosis
}

\author{
Nadezda Lachej $\mathbb{D}^{1,2}$ Daiva Dabkeviciene ${ }^{1}{ }^{2,3}$ Julija Simiene ${ }^{(D)},{ }^{2,3}$ Rasa Sabaliauskaite ${ }^{2}{ }^{2}$ \\ Violeta Jonusiene (iD, ${ }^{3}$ Vytautas Brasiunas (D), ${ }^{2}$ Ausra Sasnauskiene $\mathbb{D}^{3}{ }^{3}$ \\ Ieva Vaicekauskaite $\mathbb{D}^{2,3}$ Birute Brasiuniene $\mathbb{D}^{1,2}$ Daiva Kanopiene ${ }^{10}{ }^{2}$ \\ Kestutis Suziedelis $\mathbb{D}^{2,3}$ and Janina Didziapetriene $\mathbb{C}^{2}$ \\ ${ }^{1}$ Clinic of Internal Diseases, Family Medicine and Oncology, Institute of Clinical Medicine, Faculty of Medicine, \\ Vilnius University, LT-10257 Vilnius, Lithuania \\ ${ }^{2}$ National Cancer Institute, LT-08660 Vilnius, Lithuania \\ ${ }^{3}$ Institute of Biosciences, Life Sciences Center, Vilnius University, LT-08412 Vilnius, Lithuania
}

Correspondence should be addressed to Nadezda Lachej; nadezda.lachej@nvi.lt

Received 11 October 2021; Accepted 24 December 2021; Published 30 January 2022

Academic Editor: Ashok Pandurangan

Copyright (c) 2022 Nadezda Lachej et al. This is an open access article distributed under the Creative Commons Attribution License, which permits unrestricted use, distribution, and reproduction in any medium, provided the original work is properly cited.

New molecular biomarkers that could have an independent prognostic value in endometrial cancer are currently under investigation. Recently, it was suggested that genetic changes in the Notch signaling pathway could be associated with the development of endometrial carcinoma. This study aimed to determine the expression of the Notch signaling pathway components in tumour and adjacent normal uterine tissue and to evaluate their importance for the survival of uterine cancer patients. The present study was performed on uterine body samples collected from 109 patients and paired adjacent noncancerous endometrial tissue samples. Kaplan-Meier curves and Cox regression were used for survival analyses. Expression alterations of NOTCH2, NOTCH3, NOTCH4, $J A G 2$, and HES1 were evaluated as independent and significant prognostic factors for uterine cancer patients.

\section{Introduction}

Uterine cancer is the sixth most common cancer among women worldwide, counting about 417000 new cases and 97 000 deaths in 2020 [1]. It is an actual problem in Lithuania since approximately 600-700 cases of uterine cancer are diagnosed in Lithuania every year. It is the third most common malignancy in women after skin (except melanoma) and breast cancer and leading cancer among gynecological cancers in Lithuania [2]. Incidence rates of uterine cancer are increasing globally, primarily because of increased obesity, a significant risk factor for this disease. Other risk factors include growing life expectancy, reduced fertility, and hormone replacement therapy, especially without progestin [3]. The stage of the disease determines treatment options and has a strong influence on patient survival rates. Among women diagnosed at early stages, the 5-year survival rate is almost $82 \%$ [4]. In the diagnosis of distant metastases, the 5-year overall survival rate for advanced-stage uterine cancer is only about $25 \%$ [5].

The individualized treatment of uterine body cancer is usually based on classical clinical-pathological characteristics such as histological subtype, stage of the disease, myometrial invasion, lymphovascular invasion, and tumour differentiation grade [6]. Malignancies of the body of the uterus can be broadly classified into epithelial malignancies (endometrial carcinomas), mesenchymal malignancies (uterine sarcomas), mixed epithelial and mesenchymal malignancies of the uterus (carcinosarcomas, adenosarcomas, and carcinofibromas), and trophoblastic malignancies [7].

The tumour subtype is an important prognostic factor for the disease outcome, whereas nonendometrioid tumours are of poorer prognosis than the endometrioid subtype [8]. 
The stage of uterine cancer is associated with increasingly worse survival for the higher stages. Moreover, the deep myometrial invasion, lymphovascular space invasion, and poorly differentiated tumours are factors indicating an increased risk of recurrence and metastasis [6].

Nowadays, classical clinical-pathological characteristics seem insufficient to avoid adjuvant therapy and associated toxicity in patients with a favorable prognosis and, on the other hand, to consider additional treatment modalities to improve survival outcomes in patients with a high risk of a relapse. New molecular biomarkers that could have an independent prognostic value in endometrial cancer are currently under investigation [9].

Genetic changes in the Notch signaling pathway could be associated with the development of endometrial carcinoma [10-12]. Notch signaling is mediated by four Notch receptors (NOTCH1-4) and five transmembrane ligands (jagged 1 and jagged 2 (JAG1 and JAG2), delta-like 1 (DLL1), DLL3, and DLL4), which are called "canonical" ligands [13]. Notch receptors are single-pass transmembrane proteins with two distinct domains: an extracellular ligandbinding domain and an intracellular mediating the signal transduction [14]. After Notch receptor activation, the receptor is cleaved, and the intracellular fragment transposes to the nucleus of the cell, where it regulates the expression of transcription factors, such as the hairy enhancer of split (HES) and Hes-related (HEY) [13, 15]. Notch signaling pathway can regulate other target genes controlled by mTORC2, PI3K, TGF- $\beta$, NF $\kappa$ B, and HIF $1 \alpha$ pathways in the nucleus and/or cytoplasm [16]. In the past few decades, due to its functions, the Notch signaling pathway has been considered as a novel therapeutic target [17]. In cancerous tissues, Notch signaling can show tumour suppressive or oncogenic abilities. Aberrant expression of Notch signaling genes and their targets may be associated with cell differentiation, proliferation, tumorigenesis, metastasis formation, and epithelial-mesenchymal transition [18]. Despite increasing evidence indicating the crucial roles of Notch signaling genes in uterine cancer, the clinical significance remains unclear.

Our study aimed to determine the expression of the Notch signaling pathway components in tumour and adjacent normal uterine tissue and to evaluate their importance for the survival of uterine cancer patients. In this article, only results of the association of investigated Notch pathway components and survival of uterine cancer patients are presented.

\section{Materials and Methods}

2.1. Patients. The present study was performed on uterine body samples, collected from 109 patients with stages I-IV of uterine body cancer, who underwent surgery during the period 2010-2016 in National Cancer Institute (Vilnius, Lithuania). The censorship date is June 2020. All tumour samples had a paired control sample-adjacent noncancerous endometrial tissue (determined by histopathologists). Before carrying out the study, permission was obtained from the Vilnius Regional Biomedical Research Ethics Committee
TABLE 1: Clinical-pathological data of patients participating in the study.

\begin{tabular}{lc}
\hline Clinical-pathological characteristics & Number of patients (\%) \\
\hline FIGO stage & $51(46.8)$ \\
IA & $33(30.3)$ \\
IB & $8(7.3)$ \\
II & $1(0.9)$ \\
IIIA & $1(0.9)$ \\
IIIB & $9(8.3)$ \\
IIIC & $6(5.5)$ \\
IVB & \\
\hline The histologic type of tumour & $90(82.6)$ \\
Endometrioid adenocarcinoma & $9(8.3)$ \\
Serous adenocarcinoma & $10(9.2)$ \\
Carcinosarcoma & $38(34.9)$ \\
\hline Tumour differentiation grade & $38(34.9)$ \\
G1 & $28(25.7)$ \\
G2 & $5(4.6)$ \\
G3 & \\
Undetermined grade & $11(10.1)$ \\
\hline Metastasis to regional lymph nodes & $98(89.9)$ \\
Yes & $18(16.5)$ \\
No & $91(83.5)$ \\
\hline Lymphovascular invasion & \\
Yes & $51(46.8)$ \\
No & $58(53.2)$ \\
\hline Myometrial invasion & \\
$<1 / 2$ & $21(19.3)$ \\
$\geq 1 / 2$ & $79(72.5)$ \\
\hline Menopausal status & \\
Premenopausal & \\
\hline Body mass index & \\
$18.5-24.9$ & $(92.7)$ \\
$25.0-29.9$ & \\
$\geq 30$ & \\
\hline & \\
\hline
\end{tabular}

(protocol no. I-2010-1, issue no. 158200-05-180-43). All samples were collected with the patients' written consent to participate in the study.

All the patients in the study underwent surgical treatment: removal of the uterus (hysterectomy), including adnexa of the uterus, and pelvic and para-aortic lymphadenectomy according to indications. The mean age of the women included in the study was $65.2 \pm 9.2$ years (range 43-81). The majority of patients were 60-69 years old and $70-79$ years old $-33.9 \%$ of each group $(n=37)$; the age group $50-59-23.9 \%(n=26)$. The smallest proportion of subjects were women aged $40-49(3.7 \%, n=4)$ and $80-89$ years $(4.6 \%, n=5)$. Clinical-pathological characteristics of patients are presented in Table 1.

Depending on the stage of the disease and the degree of tumour differentiation, some patients $(n=56 ; 51.4 \%)$ underwent postoperative adjuvant radiotherapy according to the National Cancer Institute standards of treatment: some with vaginal brachytherapy $(n=28)$, others with combined radiation therapy, i.e., pelvic external-beam radiation therapy along with vaginal brachytherapy $(n=28)$. A single dose (SD) of pelvic external-beam radiation therapy was 


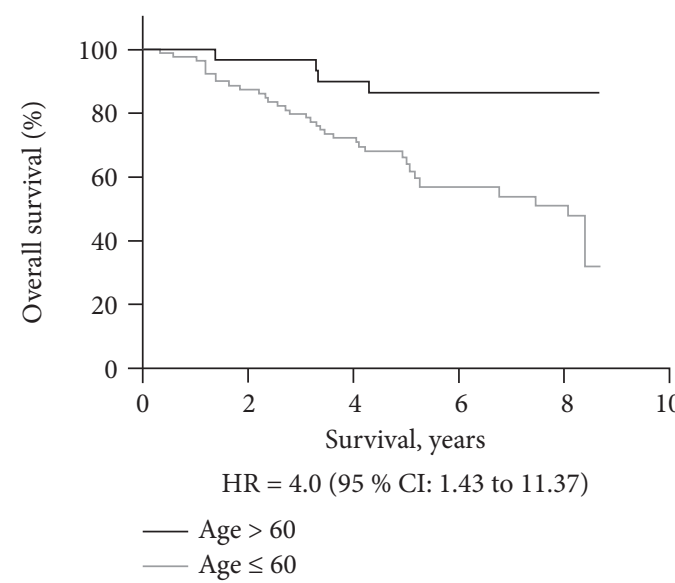

(a)

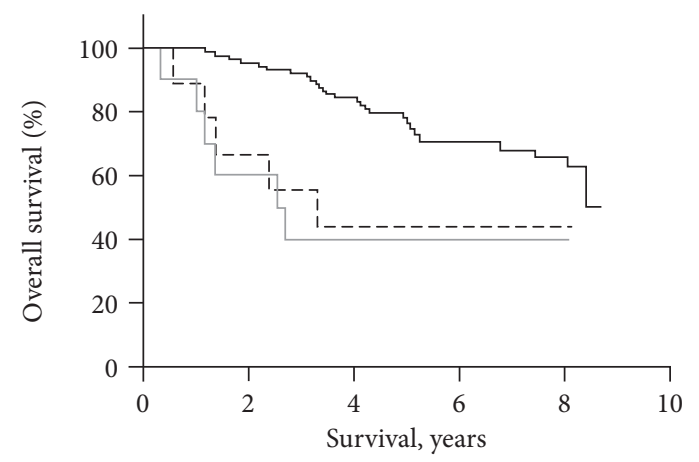

CS HR $=3.8$ (95 \% CI: 1.55 to 9.25$)$

SAC HR $=3.5$ (95\% CI: 1.31 to 9.04$)$

- EAC

CS

$---S A C$

(c)

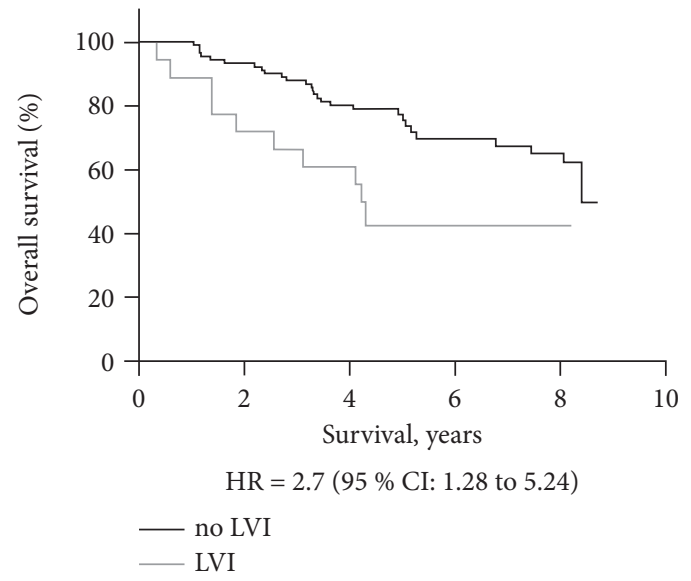

(e)

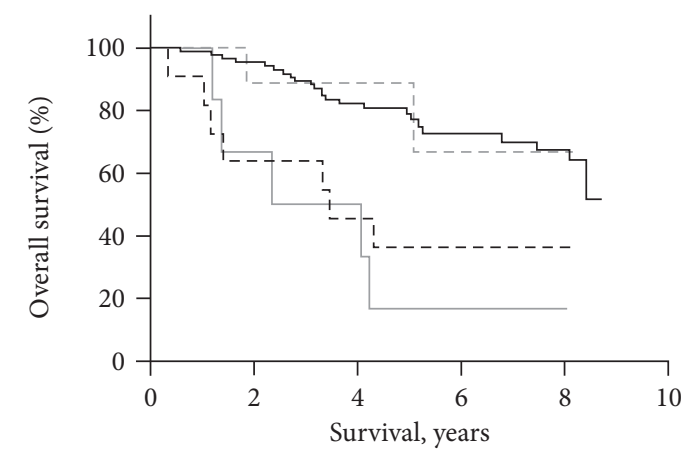

Stage II HR $=0.9$ (95 \% CI: 0.21 to 3.84$)$

Stage III HR $=3.6(95 \%$ CI: 1.55 to 8.46$)$

Stage IV HR $=5.1(95 \%$ CI: 1.93 to 13.58$)$

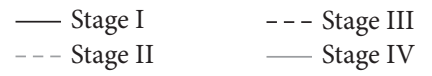

(b)

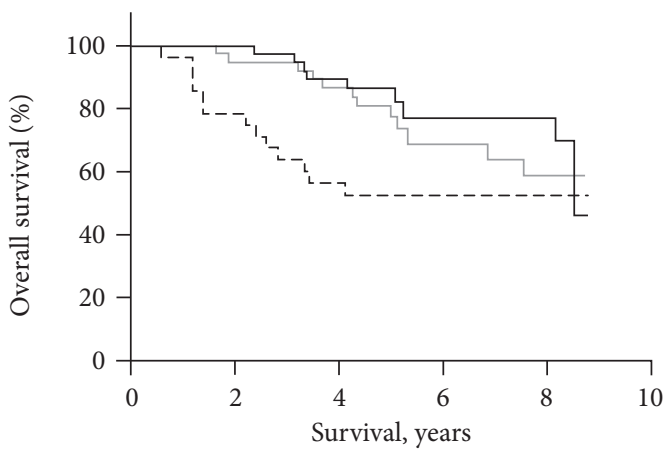

G2 HR $=1.4$ (95 \% CI: 0.58 to 3.23$)$

$\mathrm{G} 3 \mathrm{HR}=2.8$ (95\% CI: 1.21 to 6.63$)$

- G1

- G2

$---\mathrm{G} 3$

(d)

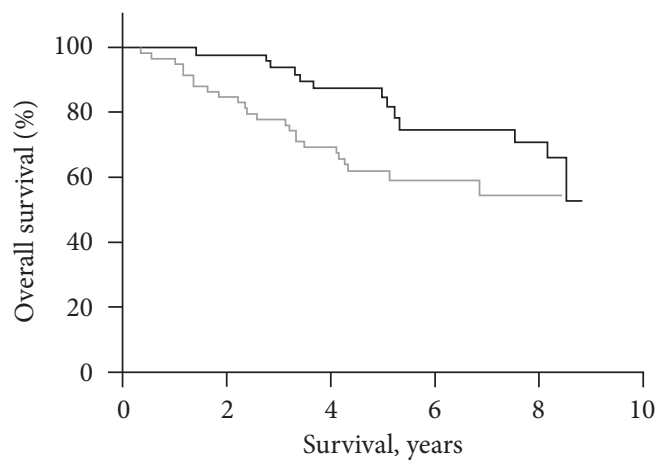

$\mathrm{HR}=2.2$ (95\% CI: 1.08 to 4.33$)$

— surgery

post-operative treatment

(f)

FIGURE 1: Overall survival rates of uterine cancer patients depending on clinical-pathological characteristics. Survival rates were dependent on as follows: age (a); the stage of the disease (b); the histologic type of tumour: EAC, endometrioid adenocarcinoma; CS, carcinosarcoma; and SAC, serous adenocarcinoma (c); the degree of tumour differentiation (G1, well-differentiated tumour; G2, moderately differentiated tumour; and G3, poorly differentiated tumour) (d); lymphovascular invasion (LVI) (e); treatment (f). 
administered-1.8-2.0 Gy in 23-28 fractions five days per week, total dose (TD) 46.0-50.4 Gy. Vaginal (intracavitary) brachytherapy was applied once a week, with $5 \mathrm{~Gy}$ (SD) at $0.5 \mathrm{~cm}$ depth in the vaginal wall during each brachytherapy procedure, using an iridium-192 source. There were three procedures combined with external-beam radiation therapy (TD, $15 \mathrm{~Gy}$ ) and four procedures with brachytherapy alone (TD, $20 \mathrm{~Gy}$ ).

Chemotherapy with cisplatin $\left(50 \mathrm{mg} / \mathrm{m}^{2}\right)$ or carboplatin dose of AUC 5 (area under the curve) in combination with doxorubicin $\left(50 \mathrm{mg} / \mathrm{m}^{2}\right)$ was administered to $16(14.7 \%)$ patients: five postoperative patients received palliative chemotherapy alone for distant metastases, while the remaining patients underwent chemotherapy before or after radiation therapy.

2.2. Sample Collection and Gene Expression Technologies. Samples of normal and pathological uterine tissues were frozen in liquid nitrogen and stored at $-80^{\circ} \mathrm{C}$ temperature. The RNA extraction, copy DNA synthesis, and reverse transcription quantitative PCR were performed following the methodology reported in our previous studies $[19,20]$.

2.3. Statistical Analysis of the Data. A sample size of 92 patients was evaluated as efficient to reach $80 \%$ power of the survival test with a $30 \%$ difference between survival rates and significance level 0.05. Kaplan-Meier curves and Cox regression were used for survival analyses. Age, FIGO stage, histologic type of tumour, and tumour differentiation grade were considered as factors having a valuable influence on the outcome. Consequently, alterations of gene expression were adjusted for the same age and clinical-pathological characteristics in multivariate Cox regression using the enter method. The cutoff for gene expression values was optimized, evaluating the most significant split between survival curves by the hazard ratio (HR) with 95\% confidence intervals. Cutoff Finder is an available web application that can be accessed via the Internet (http://molpath.charite.de/ cutoff) [21]. Associations between categorical variables were evaluated by using a two-sided Chi-square test or Fisher's exact test, as appropriate. When the $P$ value was less than 0.05 , the differences were considered statistically significant. SigmaPlot 13.0 and Statistica Basic Academic 13 were used for data analysis.

\section{Results}

3.1. Uterine Patient's Overall Survival Rate Depending on Clinical-Pathological Characteristics. The analysis of uterine patient overall survival depending on clinical-pathological characteristics data showed that females younger than age 60 with uterine cancer had a significantly longer survival rate than older patients $(p=0.008$; Figure $1(\mathrm{a}))$. As expected, the disease stage significantly influenced the survival rate $(p=0.001$; Figure $1(\mathrm{~b}))$. Patients with histologically confirmed endometrioid adenocarcinoma had a better survival rate than those with other histologic forms-carcinosarcoma and serous adenocarcinoma $(p=0.002$; Figure $1(\mathrm{c}))$. The degree of tumour differentiation was a significant determinant for survival, as the lowest degree of tumour differentiation (G3) was characterized by low survival rates $(p=0.04$; Figure $1(\mathrm{~d}))$. Significantly better survival rates were evaluated for patients without lymphovascular invasion ( $p=0.009$; Figure $1(\mathrm{e})$ ). However, the myometrial invasion had no significant effect on patient survival rates ( $p=0.83)$. Our results revealed that patients who underwent surgery alone had significantly better survival rates than those receiving postoperative radiation therapy or/and chemotherapy $(p=0.03$; Figure $1(\mathrm{f}))$.

3.2. Uterine Patient's Survival Rate Depending on the Expression of the Notch Signaling Pathway Components. The study evaluated the potential influence of the investigated Notch signaling pathway components on survival rates in patients with uterine cancer (Figure 2). The analysis of the obtained data showed a statistically significant association between the survival rate of uterine cancer patients and the expression of NOTCH2 gene (Figure 2(a)). In the case of higher $\mathrm{NOTCH} 2$ gene expression, the disease prognosis is worse $(p=0.01)$. Decreased NOTCH3 gene expression had shown a trend toward better overall survival rates $(p=0.08)$ (Figure 2(b)). Changes in NOTCH4 expression in tumour tissue were significantly associated with patient survival rates (Figure 2(c)). Patients with higher NOTCH4 expression levels had worse survival rate levels $(p=0.03)$. JAG2, DLL1, and HES1 gene expression changes lower than two folds were associated with better overall survival rate $(p=0.02$ for each gene) (Figure 2(d)-2(f)).

The correlation between subgroups of the Notch signaling pathway gene expression and clinical-pathological characteristics of the patients were evaluated and are presented in Supplementary Materials. Changes in NOTCH2 expression were significantly associated with tumour differentiation grade $(p=0.03$, Table S1) while HES1 expression-with tumour histological type $(p=0.007$, Table S2).

\subsection{Multivariate Analysis of Patients with Uterine Cancer.} Multivariate Cox regression analysis was performed to adjust gene expression to age and clinical-pathological characteristics, including stage, histology, and tumour differentiation degree. Multivariate analysis identified expression alterations of NOTCH3 and NOTCH4, as a significantly independent prognostic factor for overall survival in patients with uterine cancer. Expression alterations of NOTCH2, JAG2, and HES1 were very significantly associated with more prolonged overall survival (Table 2 ). In addition, age $(p<0.01)$ and disease stage $(p<0.001)$ were evaluated as significantly negative prognostic factors for overall survival in Cox multivariate models.

\section{Discussion}

Notch signaling drives many cellular processes and identifies as an attractive therapeutic target for uterine cancer as it is essential for the endometrial change processes [22]. Therefore, a therapeutic approach that targets specific 


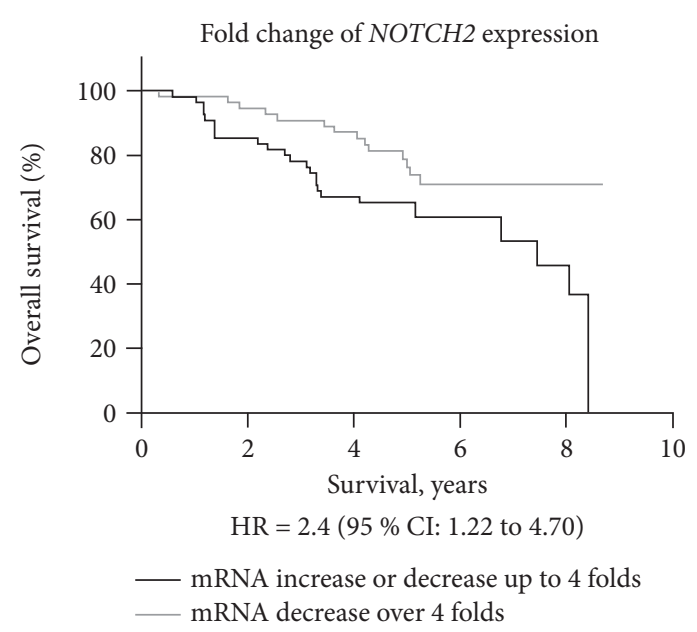

(a)

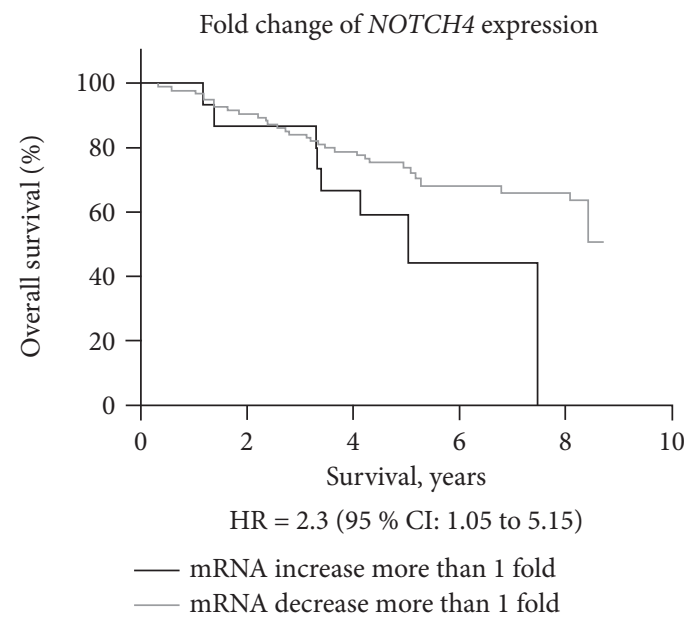

(c)

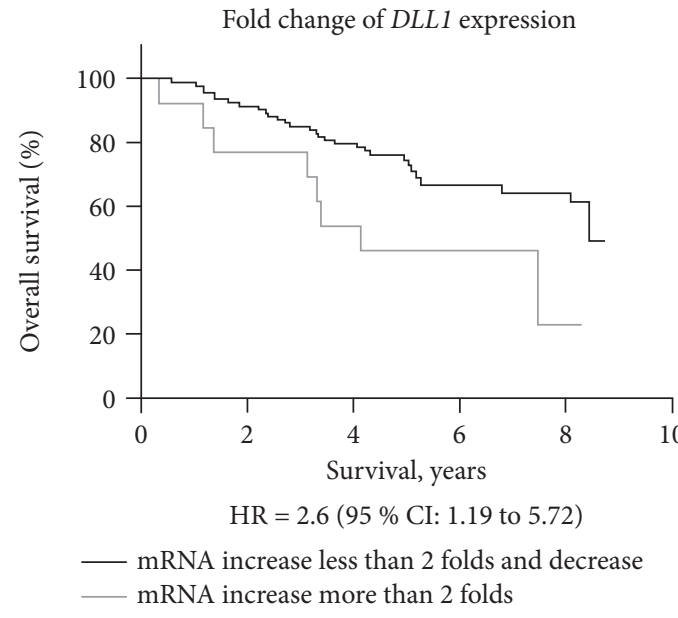

(e)

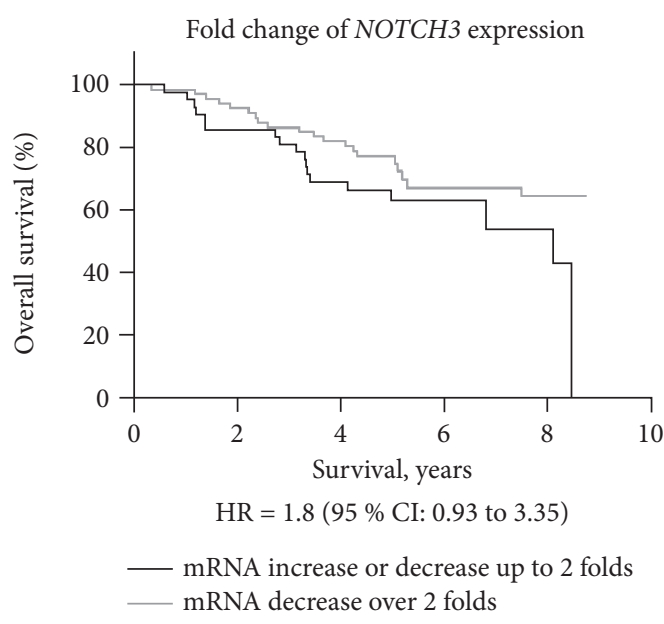

(b)

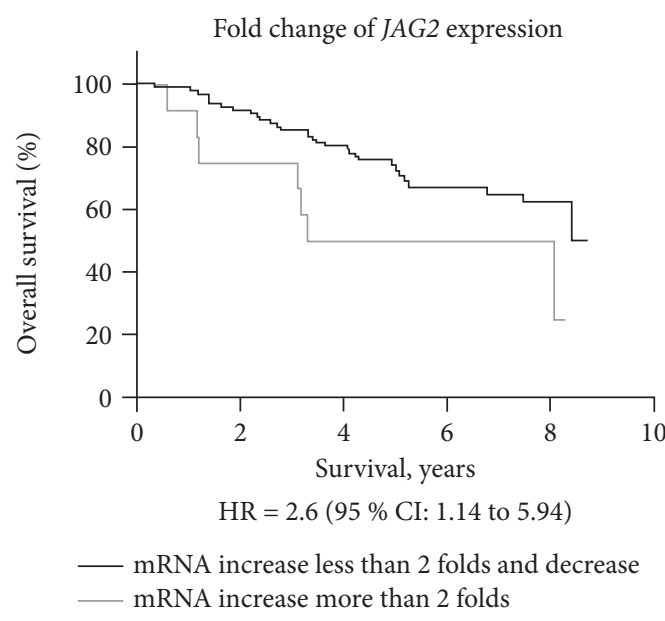

(d)

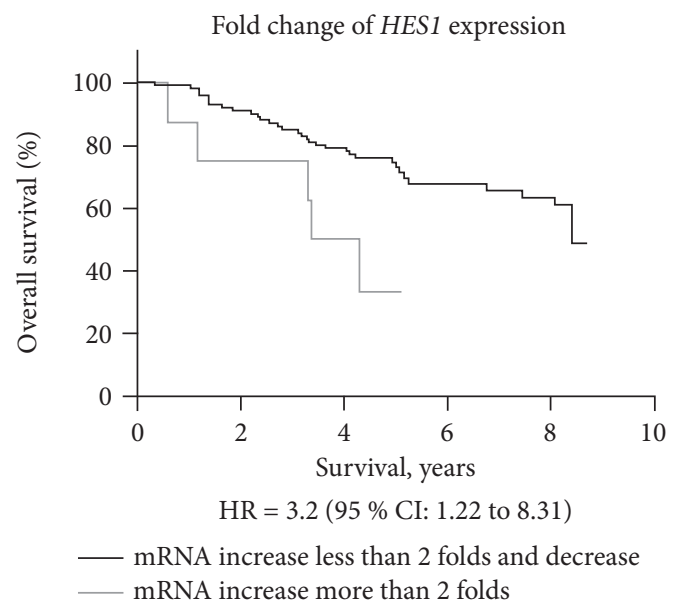

(f)

FIgURE 2: Survival rates of uterine cancer patients depending on the fold change of the NOTCH signaling pathway gene expression. NOTCH2 (a); NOTCH3 (b); NOTCH4 (c); JAG2 (d); DLL1 (e); HES1 (f).

receptors or ligands of the Notch signaling pathway that stimulate tumour cell differentiation and progression may provide more effective treatment of uterine cancer patients [23].
As expected, the analysis of our study data showed that the survival rate of patients with uterine cancer is affected by the stage of the disease, histologic type of tumour, tumour differentiation grade, and lymphovascular invasion. In our 
TABLE 2: Results of Cox multivariate models (adjustments of each gene expression to age and clinical-pathological characteristics) for overall survival of patients with uterine cancer.

\begin{tabular}{|c|c|c|c|}
\hline Gene & $p$ value & Hazard ratio & $95.0 \% \mathrm{CI}^{*}$ for hazard ratio \\
\hline $\begin{array}{l}\text { Fold change of NOTCH2 expression } \\
\text { mRNA decrease over } 4 \text { folds (reference group) } \\
\text { mRNA increase or decrease up to } 4 \text { folds }\end{array}$ & $\overline{-}$ & $\begin{array}{c}1 \\
3.4\end{array}$ & $\begin{array}{c}- \\
1.5-7.9\end{array}$ \\
\hline $\begin{array}{l}\text { Fold change of NOTCH3 expression } \\
\text { mRNA decrease over } 2 \text { folds (reference group) } \\
\text { mRNA increase or decrease up to } 2 \text { folds }\end{array}$ & $\overline{0.044}$ & $\begin{array}{c}1 \\
2.3 \\
\end{array}$ & $\frac{-}{1.0-5.1}$ \\
\hline $\begin{array}{l}\text { Fold change of NOTCH4 expression } \\
\text { mRNA decrease more than } 1 \text { fold (reference group) } \\
\text { mRNA increase more than } 1 \text { fold }\end{array}$ & $\overline{0.013}$ & $\begin{array}{c}1 \\
3.0 \\
\end{array}$ & $\frac{-}{1.3-7.3}$ \\
\hline $\begin{array}{l}\text { Fold change of JAG2 expression } \\
\text { mRNA increase less than } 2 \text { folds and decrease (reference group) } \\
\text { mRNA increase more than } 2 \text { folds }\end{array}$ & $-\overline{0.009}$ & $\begin{array}{c}1 \\
3.4\end{array}$ & $\begin{array}{c}- \\
1.4-8.5\end{array}$ \\
\hline $\begin{array}{l}\text { Fold change of HES1 expression } \\
\text { mRNA increase less than } 2 \text { folds and decrease (reference group) } \\
\text { mRNA increase more than } 2 \text { folds }\end{array}$ & $\begin{array}{c}- \\
0.009\end{array}$ & $\begin{array}{c}1 \\
4.6\end{array}$ & $\frac{-}{1.5-14.6}$ \\
\hline
\end{tabular}

${ }^{*} \mathrm{CI}$ is the confidence interval.

study, the myometrial invasion was not a statistically significant factor influencing patient survival.

The examination of the influence of Notch signaling pathway components on patient survival revealed a statistically significant association between the uterine cancer survival rate and NOTCH2-4, JAG2, and HES1 gene expression, as cases with the higher expression level of the NOTCH gene has a worse prognosis of the disease. There is limited evidence in the literature that the $\mathrm{NOTCH} 2$ receptor is an important factor in predicting the prognosis of the disease in patients with uterine cancer. In a recent publication, it has been reported that high levels of NOTCH2 expression show a statistically significant correlation with poor overall and disease-free survival time and more advanced ovarian cancer stages. In addition, $\mathrm{NOTCH} 2$, NOTCH3, DLL3, MAML1, and ADAM17 were determined as the five most relevant genes in ovarian cancer [24]. Polychronidou et al. [11] showed that higher NOTCH2 protein expression in tumour tissue might be associated with increased relapse and mortality rates of endometrial cancer patients. Also, as compared to low endometrial cancer grades, tumours with grade 3 were more frequently characterized with $\mathrm{NOTCH} 2$ and $\mathrm{NOTCH} 3$ protein overexpression.

$\mathrm{NOTCH} 3$ is an important member of the $\mathrm{NOTCH}$ family, which is involved in the development and progression of various cancers by regulating the tumour microenvironment, promoting tumour formation, angiogenesis, migration, and invasion processes $[25,26]$. It has been shown that NOTCH3 is a direct target of tumoursuppressive miR-491-5p and miR-875-5p. The activation of NOTCH3 is partly associated with the silencing of these two miRNAs. High NOTCH3 expression significantly correlates with poor survival of gastric cancer patients [27]. Moreover, multivariate Cox regression analysis of the gastric cancer patients' clinical features revealed that $\mathrm{NOTCH} 3$ expression might be used as an independent prognostic factor [28].
The data of our study for the first time demonstrate that the Notch signaling pathway receptor NOTCH4 may also play an important role in the survival of patients with uterine cancer. Results show that low NOTCH4 expression levels are associated with longer survival rates. It has been reported that NOTCH4 is implicated in cancer progression [29]. Williams et al. [10] observed the decreased expression of NOTCH4 receptor, ligand JAG1, and downstream targets HES1 and HEY1 in low-grade endometrial cancer, indicating that overall Notch signaling is suppressed in low-grade endometrial cancer. Moreover, NOTCH4 downregulation is linked to suppressed proliferation and induced apoptosis of Erbb2-negative breast cancer cell lines [30]. Another study also confirmed an association between high levels of NOTCH4 and aggressive malignant colorectal cancer cell phenotype [31]. Shawber et al. [32] identified that NOTCH4 is associated with VEGFR-3 (vascular endothelial growth factor-3), thus promoting cancer lymph node metastases. Yao et al. [33] have demonstrated that cytoplasmic NOTCH4 expression is related to Ki67 expression, suggesting that tumour cells with NOTCH4 overexpression have higher proliferation abilities.

Our study showed that patients with JAG2 gene expression change less than twofolds are associated with better overall survival rates. Contrariwise, Townsend et al. [9] demonstrated that gene expression analysis between normal and malignant patient samples showed significant elevation of the JAG2 level in endometrial cancer tissues, but it has no impact on cancer patients survival. JAG2 is associated with cell growth arresting processes due to its function as a downstream mediator of the Wnt $/ \beta$-catenin signaling pathway [34]. Chen el al. [35] showed that the NOTCH/JAG2 signaling pathway plays an important role in the regulation of bladder cancer cell proliferation, growth, and invasion processes, thus demonstrating that JAG2 expression is involved in cancer progression.

Recent studies revealed that a high HES1 expression level, which was stimulated by aberrant Notch signaling, 
correlates with increased cell proliferation in pancreatic and colon cancer [36,37]. Meanwhile, downregulation of HES1 expression is associated with decreased cell proliferation and migration abilities. Gao et al. [38] demonstrate that HES1 expression is linked to downregulation of PTEN and activation of the Akt/GSK3 $\beta$ pathway, thus enhancing the invasiveness of cancer cells.

Prognostic classification of endometrial cancer using a molecular approach based on gene panels have a potential clinical usage [39]. Testing of NOTCH components signaling in addition to other selective molecular biomarkers [40,41] may help qualify prognosis for women with uterine cancer. Our study suggests that the Notch signaling pathway, including NOTCH receptors, ligands, and target genes are essential to uterine cancer development and prognosis. Aberrant Notch signaling indicates its oncogenic potential in uterine cancer via uncontrolled cellular proliferation and avoidance of apoptosis which are two main features of cancer development.

\section{Conclusions}

Our study shows a significant role of the Notch signaling pathway components in uterine cancer. NOTCH2, NOTCH3, NOTCH4, JAG2, and HES1 may be used as independent and significant prognostic factors for uterine cancer patients.

\section{Data Availability}

The data are available on request.

\section{Ethical Approval}

All the procedures performed in studies involving human participants were in accordance with the ethical standards of the national research committee (Lithuanian Bioethics Committee, reference number 158200-05-180-43, 2010-0505).

\section{Conflicts of Interest}

The authors declare that they have no conflicts of interest.

\section{Authors' Contributions}

Nadezda Lachej and Daiva Dabkeviciene contributed equally. Nadezda Lachej, Janina Didziapetriene, Violeta Jonusiene, Ausra Sasnauskiene, and Daiva Dabkeviciene conceptualized the study. Daiva Dabkeviciene, Violeta Jonusiene, Ausra Sasnauskiene, Julija Simiene, and Nadezda Lachej designed the methodology of the study. Violeta Jonusiene, Ausra Sasnauskiene, and Julija Simiene conducted the investigation. Nadezda Lachej provided the resources. Daiva Dabkeviciene performed the data curation. Daiva Dabkeviciene, Nadezda Lachej, and Ieva Vaicekauskaite conducted the formal analysis. Nadezda Lachej, Daiva Dabkeviciene, Vytautas Brasiunas, Julija Simiene, Rasa Sabaliauskaite, Ieva Vaicekauskaite, and Birute Brasiuniene write the manuscript and conducted the literature search and analysis. Nadezda Lachej, Daiva Dabkeviciene, Kestutis Suziedelis, and Janina Didziapetriene performed the visualization. Kestutis Suziedelis and Janina Didziapetriene supervised the study. Janina Didziapetriene and Nadezda Lachej acquired the fund for the study. All the authors read and approved the final manuscript.

\section{Acknowledgments}

This project has received funding from the European Social Fund (project no. 09.3.3-ESFA-V-711-01-0001) under grant agreement with the Research Council of Lithuania (LMTLT). The authors are grateful to the patients, investigators, and staff members who participated in this study. All authors had the final responsibility for the decision to submit for publication.

\section{Supplementary Materials}

Table S1 and Table S2 are provided as separate word files. (Supplementary Materials)

\section{References}

[1] H. Sung, J. Ferlay, R. L. Siegel et al., "Global cancer statistics 2020: globocan estimates of incidence and mortality worldwide for 36 cancers in 185 countries," A Cancer Journal for Clinicians, vol. 32, 2020.

[2] Lithuanian Cancer Registry. Available at: https://www.nvi.lt/ naujausi-duomenys/.

[3] S. J. Henley, J. W. Miller, N. F. Dowling, V. B. Benard, and L. C. Richardson, "Uterine cancer incidence and mortality United States, 1999-2016," MMWR. Morbidity and Mortality Weekly Report, vol. 67, no. 48, pp. 1333-1338, 2018.

[4] W. I. u. Jayawickcrama and C. Abeysena, "Risk factors for endometrial carcinoma among postmenopausal women in Sri Lanka: a case control study," BMC Public Health, vol. 19, no. 1, p. 1387, 2019.

[5] Y. Mori, K. Yamawaki, T. Ishiguro et al., "ALDH-dependent glycolytic activation mediates stemness and paclitaxel resistance in patient-derived spheroid models of uterine endometrial cancer," Stem Cell Reports, vol. 13, no. 4, pp. 730-746, 2019.

[6] N. Singh, L. Hirschowitz, R. Zaino et al., "Pathologic prognostic factors in endometrial carcinoma (other than tumor type and grade)," International Journal of Gynecological Pathology, vol. 38, no. Suppl 1, pp. S93-S113, 2019.

[7] W. Creasman, F. Odicino, P. Maisonneuve et al., "Carcinoma of the corpus uteri," International Journal of Gynecology \& Obstetrics, vol. 95, no. Suppl 1, pp. S105-S143, 2006.

[8] T. Y. Malik, U. Chishti, A. B. Aziz, and I. Sheikh, "Comparison of risk factors and survival of type 1 and type II endometrial cancers," Pakistan Journal of Medical Sciences, vol. 32, no. 4, pp. 886-890, 2016

[9] M. H. Townsend, Z. E. Ence, A. M. Felsted et al., "Potential new biomarkers for endometrial cancer," Cancer Cell International, vol. 19, no. 1, p. 19, 2019.

[10] E. Williams, A. Villar-Prados, J. Bowser, R. Broaddus, and A. B. Gladden, "Loss of polarity alters proliferation and differentiation in low-grade endometrial cancers by disrupting Notch signaling," PLoS One, vol. 12, no. 12, Article ID e0189081, 2017. 
[11] G. Polychronidou, V. Kotoula, K. Manousou et al., "Mismatch repair deficiency and aberrations in the Notch and Hedgehog pathways are of prognostic value in patients with endometrial cancer," PLoS One, vol. 13, no. 12, Article ID e0208221, 2018.

[12] D. Daley-Brown, A. Harbuzariu, A. A. Kurian, G. Oprea-Ilies, and R. R. Gonzalez-Perez, "Leptin-induced Notch and IL-1 signaling crosstalk in endometrial adenocarcinoma is associated with invasiveness and chemoresistance," World Journal of Clinical Oncology, vol. 10, no. 6, pp. 222-233, 2019.

[13] C. S. Nowell and F. Radtke, "Notch as a tumour suppressor," Nature Reviews Cancer, vol. 17, no. 3, pp. 145-159, 2017.

[14] K. E. Vinson, D. C. George, A. W. Fender, F. E. Bertrand, and G. Sigounas, "The Notch pathway in colorectal cancer," International Journal of Cancer, vol. 138, no. 8, pp. 1835-1842, 2016.

[15] C. Shang, B. Lang, and L.-r. Meng, "Blocking NOTCH pathway can enhance the effect of EGFR inhibitor through targeting CD133+ endometrial cancer cells," Cancer Biology \& Therapy, vol. 19, no. 2, pp. 113-119, 2018.

[16] M. Janghorban, L. Xin, J. M. Rosen, and X. H. F. Zang, "Notch signalling as a regulator of the tumor immune response: to target or not to target?" Frontiers in Immunology, vol. 9, no. 1649, 2018.

[17] L. Liu, L. Zhang, S. Zhao et al., "Non-canonical notch signaling regulates actin remodeling in cell migration by activating PI3K/AKT/Cdc42 pathway," Frontiers in Pharmacology, vol. 10, p. 370, 2019.

[18] L. Li, P. Tang, S. Li et al., "Notch signaling pathway networks in cancer metastasis: a new target for cancer therapy," Medical Oncology, vol. 34, no. 10, p. 180, 2017.

[19] V. Jonusiene, A. Sasnauskiene, N. Lachej et al., "Down-regulated expression of Notch signaling molecules in human endometrial cancer," Medical Oncology, vol. 30, no. 1, p. 438, 2013.

[20] N. Lachej, V. Jonušienè, A. Mažeikè et al., "Changes in the expression of Notch and Wnt signalling molecules in human endometrial cancer," Acta Medica Lituanica, vol. 26, no. 3, pp. 181-190, 2019.

[21] J. Budczies, F. Klauschen, B. V. Sinn et al., "Cutoff Finder: a comprehensive and straightforward Web application enabling rapid biomarker cutoff optimization," PLoS One, vol. 7, no. 12, Article ID e51862, 2012.

[22] A. Sasnauskienė, V. Jonušienè, A. Krikštaponienè et al., "NOTCH1, NOTCH3, NOTCH4, and JAG2 protein levels in human endometrial cancer," Medicina, vol. 50, no. 1, pp. 14-18, 2014.

[23] S. Kumar, R. K. Srivastav, D. W. Wilkes et al., "Estrogendependent DLL1-mediated Notch signaling promotes luminal breast cancer," Estrogen-dependent DLL1-Mediated Notch Signaling Promotes Luminal Breast Cancer, vol. 23, 2014.

[24] D. Jia, J. Underwood, Q. Xu, and Q. Xie, "NOTCH2/ NOTCH3/DLL3/MAML1/ADAM17 signaling network is associated with ovarian cancer," Oncology Letters, vol. 17, no. 6, pp. 4914-4920, 2019.

[25] Q. Huang, J. Li, J. Zheng, and A. Wei, “The carcinogenic role of the notch signaling pathway in the development of hepatocellular carcinoma," Journal of Cancer, vol. 10, no. 6, pp. 1570-1579, 2019.

[26] X. Zhang, H. Shi, J. Yao et al., "FAM225A facilitates colorectal cancer progression by sponging miR-613 to regulate NOTCH3," Cancer Medicine, vol. 9, no. 12, pp. 4339-4349, 2020.

[27] W. Kang, J. Zhang, T. Huang et al., "NOTCH3, a crucial target of miR-491-5p/miR-875-5p, promotes gastric carcinogenesis by upregulating PHLDB2 expression and activating Akt pathway," Oncogene, vol. 40, no. 9, pp. 1578-1594, 2021.

[28] Y. Cui, Q. Li, W. Li et al., "NOTCH3 is a prognostic factor and is correlated with immune tolerance in gastric cancer," Frontiers in Oncology, vol. 10, Article ID 574937, 2021.

[29] Y. Sun, W. Lowther, K. Kato et al., "Notch4 intracellular domain binding to Smad3 and inhibition of the TGF- $\beta$ signaling," Oncogene, vol. 24, no. 34, pp. 5365-5374, 2005.

[30] N. Yamaguchi, T. Oyama, E. Ito et al., "NOTCH3 signaling pathway plays crucial roles in the proliferation of ErbB2negative human breast cancer cells," Cancer Research, vol. 68, no. 6, pp. 1881-1888, 2008.

[31] J. P. Shaik, I. O. Alanazi, A. A. K. Pathan et al., "Frequent activation of notch signaling pathway in colorectal cancers and its implication in patient survival outcome," JAMA Oncology, vol. 2020, Article ID 6768942, 11 pages, 2020.

[32] C. J. Shawber, Y. Funahashi, E. Francisco et al., "Notch alters VEGF responsiveness in human and murine endothelial cells by direct regulation of VEGFR-3 expression," Journal of Clinical Investigation, vol. 117, no. 11, pp. 3369-3382, 2007.

[33] K. Yao, P. Rizzo, P. Rajan et al., "Notch-1 and notch-4 receptors as prognostic markers in breast cancer," International Journal of Surgical Pathology, vol. 19, no. 5, pp. 607-613, 2011.

[34] V. Vaish, J. Kim, and M. Shim, "Jagged-2 (JAG2) enhances tumorigenicity and chemoresistance of colorectal cancer cells," Oncotarget, vol. 8, no. 32, pp. 53262-53275, 2017.

[35] Y.-T. Chen, C.-R. Huang, C.-L. Chang et al., "Jagged2 progressively increased expression from Stage I to III of Bladder Cancer and Melatonin-mediated downregulation of Notch/ Jagged2 suppresses the Bladder Tumorigenesis via inhibiting PI3K/AKT/mTOR/MMPs signaling," International Journal of Biological Sciences, vol. 16, no. 14, pp. 2648-2662, 2020.

[36] E. V. Abel, E. J. Kim, J. Wu et al., "The Notch pathway is important in maintaining the cancer stem cell population in pancreatic cancer," PLoS One, vol. 9, no. 3, Article ID e91983, 2014.

[37] M. Weng, P. Tsao, H. Lin et al., "Hes1 increases the invasion ability of colorectal cancer cells via the STAT3-MMP14 pathway," PLoS One, vol. 10, no. 12, Article ID e0144322, 2015.

[38] F. Gao, W. Huang, Y. Zhang et al., "Hes1 promotes cell proliferation and migration by activating Bmi-1 and PTEN/ Akt/GSK3 $\beta$ pathway in human colon cancer," Oncotarget, vol. 6, no. 36, pp. 38667-38680, 2015.

[39] R. López-Reig, A. Fernández-Serra, I. Romero et al., "Prognostic classification of endometrial cancer using a molecular approach based on a twelve-gene NGS panel," Scientific Reports, vol. 9, no. 1, Article ID 18093, 2019.

[40] S. R. Kim, B. T. Cloutier, S. Leung et al., "Molecular subtypes of clear cell carcinoma of the endometrium: opportunities for prognostic and predictive stratification," Gynecologic Oncology, vol. 158, no. 1, pp. 3-11, 2020.

[41] E. Coll-de la Rubia, E. Martinez-Garcia, G. Dittmar, A. GilMoreno, S. Cabrera, and E. Colas, "Prognostic biomarkers in endometrial cancer: a systematic review and meta-analysis," Journal of Clinical Medicine, vol. 9, no. 6, p. 1900, 2020. 